\title{
A New Definition of Dental Sleep Medicine
}

Frank Lobbezoo, DDS, PhD¹, Ghizlane Aarab, DDS, PhD¹, Peter Wetselaar, DDS, PhD¹, Aarnoud Hoekema MD, DMD, PhD ${ }^{1,2,3}$, Jan de Lange, MD, DDS, $\mathrm{PhD}^{4}$ \& Nico de Vries, MD, PhD ${ }^{1,5,6}$

\begin{abstract}
${ }^{1}$ Department of Oral Kinesiology, Academic Centre for Dentistry Amsterdam (ACTA), University of Amsterdam and Vrije Universiteit Amsterdam, Amsterdam, The Netherlands, ${ }^{2}$ Department of Oral and Maxillofacial Surgery, Tjongerschans Hospital, Heerenveen, The Netherlands, ${ }^{3}$ Department of Oral and Maxillofacial Surgery, University Medical Groningen Hospital, Groningen, The Netherlands, ${ }^{4}$ Department of Oral and Maxillofacial Surgery, Academic Medical Center (AMC) and Academic Centre for Dentistry Amsterdam (ACTA), University of Amsterdam and Vrije Universiteit Amsterdam, Amsterdam, The Netherlands, ${ }^{5}$ Dept. of Otorhinolaryngology, Head and Neck Surgery OLVG West, Amsterdam, The Netherlands, ${ }^{6}$ Faculty of Medicine and Health Sciences. Dept. of Otorhinolaryngology, Head and Neck Surgery, Antwerp University Hospital (UZA), Antwerp, Belgium
\end{abstract}

\begin{abstract}
Dental Sleep Medicine is the discipline concerned with the study of the oral and maxillofacial causes and consequences of sleep-related problems. Within the framework of this newly proposed definition, the discipline covers the following conditions: orofacial pain; oral moistening disorders (oral dryness, hypersalivation); gastro-esophageal reflux disorder; sleep-related breathing disorders (snoring, obstructive sleep apnea); and mandibular movement disorders (dyskinesia, dystonia, sleep bruxism). This article briefly outlines these conditions, with particular emphasis on whether they have adverse effects on patients, and when a condition should be regarded as a disorder and therefore diagnosed and if necessary managed. This is found to be the case with virtually all these conditions, except with sleep bruxism, which does not have adverse effects on everyone, in which case it counts as 'behavior'. Diagnosing and managing behavior is controversial, especially since sleep bruxism also appears to have positive effects. A cautious approach is therefore called for.
\end{abstract}

Keywords: dentistry, sleep medicine, disorder, behavior, multidisciplinary

Citation: Lobbezoo, F, Aarab G, Wetselaar P, et al. A new definition of dental sleep medicine. J Dent Sleep Med. 2018;5(4):109-112.

\section{INTRODUCTION}

Dental Sleep Medicine, an offshoot of Dentistry and more specifically of Oral Medicine, is highly multidisciplinary and requires a lot of specialist medical knowledge on the part of those working in it, including on ear, nose and throat medicine, neurology, lung disease, and internal medicine. This challenging discipline is practiced mainly by maxillofacial surgeons, orthodontists, and dentists specialized in oral medicine, dental sleep medicine, or orofacial pain and dysfunction.

Dental Sleep Medicine is an up-and-coming discipline, as is reflected both nationally and internationally in the founding of professional scientific associations (e.g., European Academy of Dental Sleep Medicine - EADSM; American Academy of Dental Sleep Medicine - AADSM), the recent publication of specialized textbooks (1), and even a peer-reviewed scientific journal (the Journal of Dental Sleep Medicine). The AADSM came up with a definition of the discipline in 2008: 'Dental Sleep Medicine focuses on the management of sleep-related breathing disorders (SBD), which includes snoring and obstructive sleep apnea (OSA), with oral appliance therapy (OAT) and upper airway surgery. ${ }^{, 1}$ Dental Sleep Medicine covers more than just breathing disorders, however, and the authors of this article therefore propose the following definition:

'Dental Sleep Medicine is the discipline concerned with the study of the oral and maxillofacial causes and consequences of sleep-related problems.'

This broadens the subject area to other problems where dentistry can (or should) play a role. Credit is due to Lavigne et al. (2), who produced a publication on this discipline almost two decades ago. In their view, Dental Sleep Medicine covers the following disorders:

- Orofacial pain

- Oral moistening disorders (including oral dryness and hypersalivation)

- Gastro-esophageal reflux disorder (GERD)

- Sleep-related breathing disorders (including snoring and OSA)

- Mandibular movement disorders (including dyskinesia, dystonia and sleep bruxism)

These disorders are briefly outlined below. Details can be found in textbooks like "Sleep Medicine for Dentists: A Practical Overview" (1) and "Principles and Practice of Sleep Medicine" (3). Only for specific details, references are provided. 


\section{OROFACIAL PAIN}

There are many types of orofacial pain: it can be dento-alveolar (i.e., due to problems with the teeth and the surrounding supporting tissue), musculoskeletal (due to muscle or joint problems), or neuropathic (due to nerve tissue problems). Headache is also classified as a type of orofacial pain. They can all affect sleep, for example by making it difficult to fall asleep or by causing nocturnal waking. All this can result in waking unrested, lowered energy levels, fatigue, lowered resistance, irritated bowels, and even feelings of depression. Nor is it the case that orofacial pain causing sleep problems is a one-way process; poor sleep can conversely cause orofacial pain. The precise relationship between the two phenomena differs from one person to another. For an overview, see De Leeuw \& Klasser (4).

\section{ORAL MOISTENING DISORDERS}

This category includes oral dryness and hypersalivation. For an overview, see Lavigne et al. (2).

\section{Oral dryness}

Oral dryness can be an actual dryness of the oral cavity due to for instance hyposalivation or the feeling of a dry mouth. The latter condition is coined "xerostomia" $(5,6)$. The unpleasant feeling of dryness is experienced in the oral cavity and throat. Its prevalence is substantial: about a quarter of the population suffer from oral dryness to a greater or lesser degree at some point in their lives (7). The following are often identified as possible causes: mouth breathing; medical conditions such as diabetes, Sjögren's syndrome, OSA and GERD; and medication (many drugs cause a feeling of dry mouth) (8). Oral dryness can cause repeated nocturnal waking, which patients often use to have a drink. All in all, then, oral dryness substantially disturbs normal sleep patterns.

\section{Hypersalivation}

Hypersalivation is excessive saliva production, which means that there is an imbalance between saliva production and swallowing frequency. Swallowing frequency is in fact lower during sleep than waking: a person swallows about three times an hour on average during sleep (9), whereas the frequency during waking is at least once every two minutes (10). Patients with hypersalivation often complain that the pillow is wet. The prevalence is substantial at $8 \%$, albeit lower than that of oral dryness (7). Possible causes are medical conditions such as Parkinson's disease (11), periodontal problems, poorly fitting or loose dentures and once again a whole range of drugs. Hypersalivation also causes repeated nocturnal waking, with all the adverse effects of sleepiness during the day and so on. It can also cause aspiration, resulting in coughing or even aspiration pneumonia.

\section{GASTRO-ESOPHAGEAL REFLUX DISORDER (GERD)}

Gastro-esophageal reflux disorder (GERD) is described as retrograde flow of gastric acid into the distal esophagus, larynx, pharynx, and even the oral cavity. The commonly used Montreal definition, a global evidencebased consensus definition for GERD (12), adds to this description that the reflux of stomach contents should cause troublesome symptoms and/or complications. Patients report GERD as heartburn that disturbs sleep, and they may also complain of an acid taste in the mouth. In addition, patients with GERD often report foreign body sensation, painful throat and hoarseness, and other aspecific throat complaints. The prevalence of GERD varies from $20 \%$ to $40 \%$ and is associated with increasing costs for health care (13). Further, epidemiological research shows that about $10 \%$ of the general population suffer from GERD at least once weekly (14), so it is a substantial problem. Factors that can cause GERD, as well as failure of the lower esophageal sphincter and diaphragmatic hernia, include obesity and pregnancy. GERD has substantial effects on the individual: it can cause erosive (chemical) tooth wear, resulting in sensitive teeth, and it can promote hypersalivation, possibly resulting in aspiration pneumonia. GERD is also associated with OSA and even premalignant or malignant mucosal lesions in the esophagus. For an overview, see Lavigne et al. (2).

\section{SLEEP-RELATED BREATHING DISORDERS}

This category includes snoring and obstructive sleep apnea (OSA). For an overview, see Lavigne et al. (2).

\section{Snoring}

This familiar condition is characterized by loud breathing sounds produced in the upper airway during sleep. It is a common problem, which increases with age until the point is reached where about half of men and women over the age of 65 snore regularly. Where OSA is absent the effects are mainly social, with bed partners in particular suffering from sleep disturbance, which can be severe. The effects can therefore be substantial. In load snoring individuals, a sleep study has to be considered since it is the most important alarm symptom for OSA (15).

\section{Obstructive Sleep Apnea (OSA)}

OSA is characterized by repeated obstruction of the upper airway, causing oxygen desaturation and frequent awakenings of the patient. 14-49\% of middle-aged men in the United States and Europe have clinically significant OSA (16). The etiology is multifactorial and still not fully clarified: associations have been found with various demographic, anatomical, biological and behavioral factors. The effects are substantial: OSA can cause the patient to wake 
unrested, excessive fatigue during the day, high blood pressure, cardiovascular disease, cognitive impairment, reduced libido, and a whole range of other health problems, some of them severe (including mortality). For an overview, see Lavigne et al. (2).

\section{MANDIBULAR MOVEMENT DISORDERS}

This category includes dyskinesias, dystonias and sleep bruxism. For an overview, see Michelotti et al. (17).

\section{Orofacial dyskinesias}

This group of motor dysfunctions is characterized by involuntary, mainly choreatic (dance-like) movements of the face, lips, tongue and jaws. If the condition is confined to the jaws it is important to differentiate it from bruxismrelated teeth grinding (18). Possible causes can be underlying psychiatric disorders and certain drugs (e.g., neuroleptics and dopamine-related drugs). Orofacial dyskinesia has an enormous impact on everyday life, but not enough is yet known about the local effects. Patients with this disabling condition can also have disturbed sleep.

\section{Oromandibular dystonias}

Where these excessive, involuntary, persistent muscle contractions in the lips, tongue, and jaws are confined to the latter structure, they can easily be confused with bruxism-related clenching (18). This is nevertheless a genuine and potentially disabling motor dysfunction, which has the same possible causes and effects as the orofacial dyskinesias outlined above.

\section{Sleep bruxism}

Bruxism has recently been defined as repetitive masticatory muscle activity characterized by clenching or grinding the teeth and/or bracing or thrusting the mandible (19). Although bruxism can also occur during the day (awake bruxism), here we shall briefly consider sleep bruxism, referring to the review by Lobbezoo et al. (20). The prevalence of sleep bruxism in the general population is $8 \%$. A variety of factors play a role in the multifactorial etiology: psychosocial (stress, anxiety), biological (neurotransmitter abnormalities, genetics), and exogenous (medication, smoking, alcohol). The following are identified as possible effects: breakage or loss of teeth, fillings or implants; mechanical tooth wear in the form of attrition; masticatory muscle hypertrophy; musculoskeletal pain; and mandibular dysfunctions. It should be stressed that some bruxists do not experience any of these adverse effects.

\section{DISORDER OR BEHAVIOR?}

The conditions described above are frequently referred to as 'disorders', and a disorder should by definition be diagnosed and if necessary managed. A few of the con- ditions described above could also be referred to as 'behavior', however. Behavior is only a disorder if it causes some kind of harm or is detrimental to the individual; if this is not the case it is simply behavior, and diagnosis and management are not indicated (21). Which of the conditions described above can justifiably be referred to as disorders? In effect they may all have serious effects on the individual. The only case where this does not seem to apply is snoring (without OSA): here the harm is mainly to the bed partner, if any. However, since this in turn has social consequences for the snorer, it makes snoring behavior a snoring disorder for the individual concerned, and diagnosing and if necessary managing it is then justifiable. In addition, it should again be noted that loud snoring is the most important alarm system for OSA, hence justifying additional diagnostic procedures as well. The situation is somewhat more complicated with sleep bruxism. As already said, not all bruxists experience adverse effects from their behavior. Diagnosis and management are not appropriate for them, first and foremost to avoid "overdiagnosis" and "overmanagement", but also because there are some indications that bruxism - in addition to the supposed adverse effects - can also have positive effects on the individual, such as stimulating salivation (thus preventing dry mouth during sleep) and helping to maintain adequate muscle tone in the upper airway (thus possibly preventing OSA) (22).

\section{CONCLUSION}

The newly proposed definition of Dental Sleep Medicine enables the inclusion of not only sleep-related breathing disorders into this dental discipline, but also of other conditions that may have oral and maxillofacial causes and consequences. Although the recent founding of dedicated professional Academies suggests that Dental Sleep Medicine is a genuine new offshoot of Dentistry, some will consider the discipline as part of wider discipline "Oral Medicine". However, since Oral Medicine as a dental discipline does not exist in every country, and if it does, is not always widespread, the authors of this article consider the existence of Dental Sleep Medicine as a separate dental discipline justified, the more so since many of the above outlined conditions deserve more attention from dental professionals.

Most of the conditions that fall into the category of Dental Sleep Medicine are disorders (i.e., they have adverse effects on the individual) and should therefore be diagnosed and if necessary managed. The exception is sleep bruxism, which does not always harm the patient. For this reason - and because sleep bruxism also appears to have positive effects on the individual - a cautious approach is called for when diagnosing and managing bruxism behavior. 


\section{REFERENCES}

1. Lavigne GJ, Cistulli P, Smith M (eds.). Sleep Medicine for Dentists: A Practical Overview. Chicago, Il: Quintessence Publishing Co, Inc., 2009.

2. Lavigne GJ, Goulet JP, Zuconni M, Morisson F, Lobbezoo F. Sleep disorders and the dental patient: A review of diagnosis, pathophysiology and management. Oral Surg Oral Med Oral Pathol Oral Radiol Endod. 1999;88:257-272.

3. Kryger MH, Rith T, Dement WC (eds.). Principles and Practice of Sleep Medicine, $6^{\text {th }}$ Ed. Philadelphia, PA: Elsevier Health Sciences, 2016.

4. Leeuw R de, Klasser GD (eds.). Orofacial Pain. Guidelines for Assessment, Diagnosis, and Management. The American Academy of Orofacial Pain. Chicago, IL: Quintessence Publishing Co, Inc, 2013.

5. Nederfors T. Xerostomia and hyposalivation. Adv Dent Res. 2000;14:48-56.

6. Löfgren CD, Wickström C, Sonesson M, Lagunas PT, Christersson C. A systematic review of methods to diagnose oral dryness and salivary gland function. BMC Oral Health. 2012;12:29.

7. Lalonde B, Lavigne GJ, Goulet J-P, Barbeau J. Prevalence of reported salivary dysfunction symptoms in an adult Northern population. J Dent Res. 1997;76:99.

8. Scully C. Drug effects on salivary glands: dry mouth. Oral Dis. 2003;9:165-176.

9. Sato K, Nakashima T. Human adult deglutition during sleep. Ann Otol Rhinol Laryngol. 2006;115;334-339.

10. Huizing EH, Snow GB, Vries N de, Graamans K, Heijning P van de. Keel- neus- oorheelkunde en hoofd-halschirurgie [Ear, nose \& throat medicine and head \& neck surgery]. Houten, The Netherlands: Bohn Stafleu van Loghum, 2007.

11. Kalf JG, de Swart BJ, Borm GF, Bloem BR, Munneke M. Prevalence and definition of drooling in Parkinson's disease: a systematic review. J Neurol. 2009;256:1391-1396.

12. Vakil N, Zanten SV van, Kahrilas P, Dent J, Jones R; Global Consensus Group. The Montreal definition and classification of gastroesophageal reflux disease: a global evidence-based consensus. Am J Gastroenterol. 2006; 101: 1900-1920.

13. Dent J, El-Serag HB, Wallander MA, Johansson S. Epidemiology of gastro-oesophageal reflux disease: a systematic review. Gut. 2005;54:710-717.

14. Djärv T, Wikman A, Nordenstedt H, Johar A, Lagergren J, Lagergren P. Physical activity, obesity and gastroesophageal reflux disease in the general population. World J Gastroenterol. 2012; 18:3710-3714

15. Deary V, Ellis JG, Wilson JA, Coulter C, Barclay NL. Simple snoring: not quite so simple after all? Sleep Med Rev. 2014;18:453-462.

16. Garvey JF, Pengo MF, Drakatos P, Kent BD. Epidemiological aspects of obstructive sleep apnea. J Thorac Dis. 2015;7:920-929.

17. Michelotti A, Alstergren P, Goulet JP, Lobbezoo F, Ohrbach R, Peck C, Schiffman E, List T. Next steps in development of the diagnostic criteria for temporomandibular disorders (DC/TMD): Recommendations from the 2014 International RDC/TMD Consortium Network workshop. J Oral Rehabil 2016;43:453-467.

18. Lobbezoo F, Naeije M. Dental implications of some common movement disorders: A concise review. Archs Oral Biol. 2007;52:395398.
19. Lobbezoo F, Ahlberg J, Glaros AG, Kato T, Koyano K, Lavigne GJ et al. Bruxism defined and graded: An international consensus. $\mathbf{J}$ Oral Rehabil. 2013;40;2-4.

20. Lobbezoo F, Ahlberg J, Manfredini D, Winocur E. Are bruxism and the bite causally related? J Oral Rehabil. 2012;39;489-501.

21. Raphael KG, Santiagio V, Lobbezoo F. Is bruxism a disorder or a behavior? Rethinking the International Consensus on Defining and Grading of Bruxism. J Oral Rehabil 2016;43:791-798.

22. Manfredini D, Guarda-Nardini L, Marchese-Ragona R, Lobbezoo F Theories on possible temporal relationships between sleep bruxism and obstructive sleep apnea events. An expert opinion. Sleep Breath 2015;19:1459-1465.

\section{SUBMISSION AND CORRESPONDENCE INFOR- MATION}

\author{
Submitted in final revised form June 5, 2018 \\ Accepted for publication September 14, 2018
}

Address correspondence to: Prof. Frank Lobbezoo, Department of Oral Kinesiology, Academic Centre for Dentistry Amsterdam (ACTA), Gustav Mahlerlaan 3004, 1081 LA Amsterdam, The Netherlands; Tel: +3120 5980412; Fax: +3120 5980333; Email: f.lobbezoo@acta.nl

\section{ACKNOWLEDGMENTS}

This paper was previously published in the Journal of Oral Rehabilitation (citation: Lobbezoo F, Aarab G, Wetselaar P, Hoekema A, Lange J de, Vries N de. A new definition of Dental Sleep Medicine. J Oral Rehabil 2016;43:786-790) and is republished with permission from John Wiley and Sons, Hoboken, NJ, USA. The paper is a translated and adapted version of the Dutch article 'Tandheelkundige slaapgeneeskunde: een overzicht' by F. Lobbezoo et al., published in ACTA Quality Practice, 2015, with permission from Prelum Publishers, Houten, The Netherlands.

\section{DISCLOSURE STATEMENT}

Frank Lobbezoo is Member of the Academic Advisory Board of Sunstar Suisse S.A. Aarnoud Hoekema is Medical Advisor of Zephyr Sleep Technologies. Nico de Vries is Medical Advisor of Night Balance, Philips Health care, Olympus, and ReVent, has shares in NightBalance and stock options in ReVent, and is researcher for Inspire. Ghizlane Aarab, Peter Wetselaar, and Jan de Lange report no conflicts of interest. 